\title{
Dietary quality, as measured by the Alternative Healthy Eating Index for Pregnancy (AHEI-P), in couples planning their first pregnancy
}

\author{
Pao Ying Hsiao ${ }^{1, *}$, June L Fung ${ }^{2}$, Diane C Mitchell ${ }^{3}$, Terryl J Hartman ${ }^{4}$ and \\ Marlene B Goldman ${ }^{2}$ \\ 'Department of Food and Nutrition, Indiana University of Pennsylvania, 911 South Drive, Indiana, PA 15705, USA: \\ ${ }^{2}$ Department of Obstetrics and Gynecology, Dartmouth-Hitchcock Medical Center, Geisel School of Medicine at \\ Dartmouth, Lebanon, NH, USA: ${ }^{3}$ Department of Nutritional Sciences, The Pennsylvania State University, University \\ Park, PA, USA: ${ }^{4}$ Rollins School of Public Health, Department of Epidemiology, Emory University, Atlanta, GA, USA
}

Submitted 11 July 2018: Final revision received 5 March 2019: Accepted 11 March 2019: First published online 27 May 2019

\begin{abstract}
Objective: Dietary quality (DQ), as assessed by the Alternative Healthy Eating Index for Pregnancy (AHEI-P), and conception and pregnancy outcomes were evaluated.

Design: In this prospective cohort study on couples planning their first pregnancy. Cox proportional hazards regression assessed the relationship between AHEI-P score and clinical pregnancy, live birth and pregnancy loss.

Setting: Participants were recruited from the Northeast region of the USA. Participants: Healthy, nulliparous couples (females, $n 132$; males, $n 131$; one male did not enrol).

Results: There were eighty clinical pregnancies, of which sixty-nine resulted in live births and eleven were pregnancy losses. Mean (SD) female AHEI-P was 71.0 (13.7). Of those who achieved pregnancy, those in the highest tertile of AHEI-P had the greatest proportion of clinical pregnancies; however, this association was not statistically significant $(P=0 \cdot 41)$. When the time it took to conceive was considered, females with the highest AHEI-P scores were $20 \%$ and $14 \%$ more likely to achieve clinical pregnancy (model 1: hazard ratio $(\mathrm{HR})=1 \cdot 20 ; 95 \% \mathrm{CI}$ $0 \cdot 66,2 \cdot 17$ ) and live birth (model $1: \mathrm{HR}=1 \cdot 14 ; 95 \%$ CI $0.59,2 \cdot 20$ ), respectively. Likelihood of achieving clinical pregnancy and live birth increased when the fully adjusted model, including male AHEI-P score, was examined (clinical pregnancy model 4: $\mathrm{HR}=1.55 ; 95 \% \mathrm{CI} 0.71,3.39$; live birth model 4: $\mathrm{HR}=1.36$; $95 \%$ CI 0.59, 3.13).

Conclusions: The present study is the first to examine AHEI-P score and achievement of clinical pregnancy. DQ was not significantly related to pregnancy outcomes, even after adjustments for covariates.
\end{abstract}

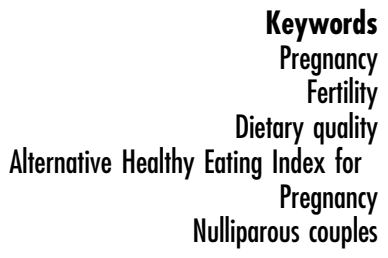

The role of dietary intake in conception is a topic of emerging interest. While eating a varied diet is a key part of overall health, certain food groups and nutrients have been shown to be beneficial in reproductive health. Recent studies have suggested that nutritional factors, including fruits, vegetables and antioxidants such as vitamins $\mathrm{C}$ and $\mathrm{E}$ and Se, may be beneficial for fertility; while other dietary components, such as trans-fatty acids, protein, alcohol and caffeine, have been associated with detrimental effects on fertility ${ }^{(1-3)}$. Interestingly, male diet has been shown to impact fertility as well ${ }^{(4-7)}$. Diets higher in carbohydrate, fibre, folate, vitamin $\mathrm{C}$ and lycopene $\mathrm{e}^{(8)}$, as well as higher in fruits and vegetables ${ }^{(9)}$, have been shown to be associated with higher sperm quality. Conversely, male intake of saturated fat ${ }^{(10)}$, trans fat ${ }^{(11)}$ and alcohol ${ }^{(12)}$ has been shown to have detrimental effects on reproductive outcomes. While a substantial body of research has focused on individual nutrients important during the preconception period, less is known about dietary patterns during this time. 
Increasingly dietary patterns, rather than just individual nutrients or foods, are being assessed to evaluate the relationship between dietary intake and health outcomes. Conceptually, dietary patterns may better represent these relationships as the methodology captures the synergistic relationship that nutrients consumed in foods may have with each other ${ }^{(13)}$. Dietary patterns can be evaluated using indices, or a summary score, that capture specific components that are associated with outcomes of interest. One such index is the Alternative Healthy Eating Index (AHEI).

The AHEI is an a priori dietary index that is based on the Healthy Eating Index, which was developed by the US Department of Agriculture ${ }^{(14)}$. The AHEI is a measure of diet quality that focuses on foods and macronutrients, including assessment of unsaturated fats, associated with decreased chronic disease risk $^{(15,16)}$. To make the AHEI suitable for use in a pregnant population, Poon and colleagues modified the score to create the Alternative Healthy Eating Index for Pregnancy (AHEI-P) by excluding the alcohol component and including components for nutrients important for pregnancy (i.e. Ca, folate and $\mathrm{Fe})^{(17)}$. The AHEI-P has been used to determine the relationship of maternal diet in the third trimester with birth weight and early infant growth ${ }^{(17)}$. Diet quality during the preconception period has not been previously assessed using the AHEI-P.

The Lifestyle and Fertility (ISIS) study, named after the Egyptian goddess of fertility, was designed and conducted specifically to prospectively evaluate the impact of measures of nutritional status on achieving pregnancy in couples planning their first pregnancy. The goal of the present analysis was to determine the association between dietary quality as assessed by the AHEI-P and achieving pregnancy in the ISIS study. Less is known about dietary quality and male fertility; therefore a secondary goal of the present study was to consider the potential role of diet quality for the male partner.

\section{Methods}

\section{Study participants}

Study participants were recruited to be in the ISIS study, a multi-site, prospective cohort study of healthy, nulliparous couples with no known infertility conditions, who were planning their first pregnancy. Participants were recruited using a variety of methods, including referrals from medical providers in both primary care and obstetrics and gynaecology practices, posting of brochures, posters and fliers in public spaces in and around surrounding areas of the sites, advertisements in local newspapers, and postcard mailings to potential participants.

Of the 802 couples who went through preliminary screening, including a baseline pregnancy test to ensure that female participants were not pregnant at the start of the study, 181 were eligible. Couples were ineligible if there was history of recognized conception, history of infertility or if the female had polycystic ovary syndrome. One hundred and thirty-two couples passed further screening and were enrolled between May 2008 and June 2012 after attending a baseline visit at one of three clinical sites in the Northeast region of the USA (Boston, MA; State College, PA; and Lebanon, NH). Females were between 18 and 39 years of age. All but one male partner was enrolled. Therefore, the study sample consisted of 132 females and 131 males. The protocol was approved by the participating institutions' institutional review boards and all participants provided written informed consent. This trial was registered with Clinicaltrials.gov (ClinicalTrials.gov identifier: NCT00642590).

\section{Protocol}

Lifestyle factors, dietary intakes and biochemical measures were assessed among the couples. The couples were followed from the start of the study protocol until they achieved a clinically confirmed pregnancy, completed six menstrual cycles of attempted conception or were lost to follow-up. Pregnancy was first determined by a positive pregnancy test (human chorionic gonadotrophin $\geq 20 \mathrm{mIU} / \mathrm{ml}$; AIM MidStream OTC Professional, Craig Medical Distribution, Inc., Vista, CA, USA) and confirmed clinically by each female participant's physician by a urine test, blood test and/or ultrasound. Those who conceived a clinical pregnancy were followed through the delivery or loss of the pregnancy.

\section{Dietary intake}

Dietary intakes were assessed at baseline using a series of three (two weekday and one weekend day) unannounced $24 \mathrm{~h}$ dietary recalls collected via telephone by trained interviewers of The Pennsylvania State University Diet Assessment Center (University Park, PA, USA). Couples were individually contacted and asked to recall all foods and beverages consumed the previous day. Participants were provided a poster of two-dimensional models to improve accuracy of portion estimation (2-D Food Portion Visual; Nutrition Consulting Enterprise, Framingham, MA, USA). A multiple-pass technique was utilized to facilitate recall as well as standardize the process $^{(18)}$. Quality control procedures included range checks for energy (kcal) and selected vitamins, in addition to two questions answered at the end of each food intake interview. One question asked participants if their intake was 'typical', 'more than usual' or 'less than usual'. The second question was the interviewer's assessment of the reliability of the participant's report of her/his intake. For example, data might be coded as 'unreliable' if the participant was unable to recall one or more meals or was unreliable for other reasons. In the current study, we had several 
days of intake marked as 'unreliable' for language barriers, but the intakes were in a normal range (e.g. $<2092 \mathrm{~kJ}(<500 \mathrm{kcal})$ and >20 $920 \mathrm{~kJ}$ (>5000 kcal)). No participants in our sample reported implausible intakes (e.g. $<2092$ or $>20920 \mathrm{~kJ}(<500$ or $>5000 \mathrm{kcal}))$. To reflect the marketplace throughout the study, dietary intake data were collected using Nutrition Data System for Research (NDSR) software versions 2008, 2009, 2010 and 2011, developed by the Nutrition Coordinating Center (NCC), University of Minnesota, Minneapolis, MN, USA. Final calculations were completed using NDSR version 2012. The NDSR time-related database updates analytic data while maintaining nutrient profiles reflective of the marketplace at the time data were collected.

The AHEI-P is a dietary quality index based on a 130point scale with 0-10 points awarded for optimal intake of six food groups (vegetables; whole fruit; whole grains; sugar-sweetened beverages; nuts and legumes; red/processed meats) and seven nutrient-based categories (trans fats; long-chain fats; PUFA; Na; Ca; folate; $\mathrm{Fe}$ ), with a higher score indicative of a higher quality diet ${ }^{(17)}$. To calculate the score for healthy components (vegetables; whole fruit; whole grains; nuts and legumes; longchain fats; PUFA; Ca; folate; Fe), intake of each food group or nutrient was divided by the criterion for maximum points and multiplied by 10 . For less healthy components (sugar-sweetened beverages; red/processed meats; trans fats; Na), intake was divided by the criterion for maximum points, subtracted by 1 , and then multiplied by 10 . This calculation allows for higher points to be assigned for lower intake of the less healthy components. Nutrient intake was calculated from 'foods and beverages only' (i.e. supplements were not included). Average intakes from baseline dietary recall data were used to calculate AHEI-P in both the males and females.

\section{Covariates}

Baseline demographic characteristics including age, race/ ethnicity, education, household income, smoking status and age at menarche were obtained through self-report by the participants at enrolment. Female participants were also asked if they had regular menstrual cycles at the time of the baseline visit (regular $v$. irregular) and their amount of weekly aerobic physical activity (<30 min; 30-59 min; $1-2 \mathrm{~h} ; \geq 3 \mathrm{~h}$ ). Dietary covariates (e.g. energy, caffeine and alcohol) were estimated from the average of the dietary recalls. In addition, participants were weighed (within $0 \cdot 1 \mathrm{~kg}$ ) without shoes and wearing only light clothing on a calibrated, digital scale (SECA, Chino, CA, USA). Standing height was measured without shoes (within $0.5 \mathrm{~cm}$ ) on a wall-mounted stadiometer. All measurements were taken twice and averaged. If the first two measurements disagreed ( $>0.5 \mathrm{~cm}$ for height or $>0.2 \mathrm{~kg}$ for weight), a third measurement was taken and the two closest were averaged and recorded. BMI was calculated for each participant as [weight $(\mathrm{kg})] /[\text { height }(\mathrm{m})]^{2}$.

\section{Pregnancy outcomes}

Each confirmed clinical pregnancy was followed to determine the outcome, including date of loss or delivery of a live birth. Live birth was defined as a gasp, heart beat or sign of life at birth. Pregnancy loss included spontaneous and therapeutic abortions.

\section{Statistical analyses}

The final analytical sample included 263 participants (females, $n$ 132; males, $n$ 131). In addition, two males did not provide dietary data and therefore were excluded from any analyses that used male dietary data. AHEI-P scores were analysed as both sex-specific tertiles and continuous variables (5-point increments). The $t$ test was used to examine statistical differences between females and males for AHEI-P components and total AHEI-P score (Table 1). ANOVA (continuous data) and $\chi^{2}$ and Fisher's exact tests (categorical data) were used to examine baseline maternal demographic, socio-economic and healthrelated characteristics by AHEI-P-tertile (Table 2). The $\chi^{2}$ test was used to analyse the association between clinical pregnancy and AHEI-P tertile (Table 3).

To account for those couples who took longer to conceive, time to conception was considered using Cox proportional hazards regression models (PROC PHREG in SAS). This method captures measurement of an event from a defined starting point to the outcome(s) of interest (e.g. clinical pregnancy). To complete this analysis, a 'time to event' variable (i.e. time to conception), which takes account of varying start and 'censoring' times, was constructed for each participant. The Cox model has advantages, namely it accounts for the presumed reduction in the probability of conception with each subsequent menstrual cycle that goes by, by building it into the baseline hazard function. Ideally, time in the Cox model would be measured as the number of menstrual cycles since baseline. While cycle length varies between females and even varies within-person, an average cycle length, often $30 \mathrm{~d}$, is used to represent the sample; however, in the absence of these data, time measured as 'days' was used in the present analyses.

Time to conception of a clinical pregnancy was defined as the length of time in 'days' from the date the couple began attempting conception (day 1 of the first menstrual cycle after the baseline visit) to the date of the last menstrual period plus $14 \mathrm{~d}$. Cox proportional hazards regression models were also used to examine 'time to conception of a clinical pregnancy resulting in a live birth' and 'time to conception of a clinical pregnancy resulting in a pregnancy loss' as events (Table 4). Those who conceived prior to the start of their first cycle were censored at the time of their 
baseline visit ( $n$ 12). For those who did not conceive a clinical pregnancy and had follow-up to determine outcome, time was censored at the end of the six menstrual cycles. Those without follow-up (i.e. staff attempted to contact them and could not reach them) were censored at the date of last contact ( $n 5$, of which $n 3$ were at the baseline visit). Those who were censored at their baseline visit ( $n$ 15) were not included in the time to conception analyses.

Models were statistically adjusted for age and energy (kcal; model 1), with additional adjustment for female BMI (model 2). Model 3 included all adjustments from model 2, in addition to male education and smoking status. Partner's education, in this case the male partner, is functioning as a marker for both health behaviours and economic status. Partner's education has been significantly associated with self-assessed health ${ }^{(19)}$. Lastly, male dietary quality, as measured by AHEI-P score (model 4), was included as a covariate. In a series of sensitivity analyses, we evaluated whether either caffeine intake or alcohol consumption was an important contributor in multivariate models. Finally, we examined male AHEI-P score as the exposure of interest (online supplementary material). All models are reported with their respective estimates and 95\% CI.

Assumptions of the Cox proportional hazards regression model were tested (e.g. examination of survival plots) and met. Statistical significance was set at $P<0.05$. All data analyses were performed using the statistical software package SAS version 9.4 (University Edition, 2014).

\section{Results}

Baseline mean (SD) AHEI-P scores are presented in Table 1. Males and females showed statistically significant differences in mean AHEI-P component scores for whole fruit, red/processed meat, trans fats, $\mathrm{Na}, \mathrm{Ca}$, folate and Fe $(P<0.05)$; however, total AHEI-P score was not significantly different between males and females $(P=0 \cdot 19)$. AHEI-P mean (SD) tertile scores for females were 55.5 (6.2), 71.0 (4.1) and 86.4 (5.6) for T1 (range = 42.5-65.0), T2 (range $=65 \cdot 2-78 \cdot 1)$ and T3 (range $=78 \cdot 6-102 \cdot 0)$, respectively. When examined by AHEI-P tertile score, females showed no statistically significant differences in baseline characteristics across tertiles, with the exception of education and pre-pregnancy (baseline) BMI. Females with lower AHEI-P scores tended to have higher prepregnancy BMI. When examined by female AHEI-P tertile score, males showed significant differences for education, smoking status, BMI and AHEI-P score (Table 2). Male partners of females with lower AHEI-P scores tended to have lower educational status and higher BMI.

There were eighty clinical pregnancies, of which sixtynine resulted in live births and eleven were pregnancy losses, reported in the present study. Of those who achieved clinical pregnancy, those in the highest tertile of AHEI-P had the greatest proportion of clinical pregnancies; however, this association was not found to be significant $(P=0 \cdot 41$; Table 3$)$.

When time to conception was considered as an outcome, using Cox proportional hazards regression modelling on a sample of 117 couples, females with the highest AHEI-P scores were $20 \%$ and $14 \%$ more likely to achieve clinical pregnancy (model 1: hazard ratio $(\mathrm{HR})=1.20 ; 95 \%$ CI $0.66,2.17)$ and live birth (model 1 : $\mathrm{HR}=1 \cdot 14 ; 95 \%$ CI $0 \cdot 59,2 \cdot 20$ ), respectively. Likelihood of achieving clinical pregnancy and live birth increased when the fully adjusted model, including male AHEI-P score, was examined (clinical pregnancy model 4 : $\mathrm{HR}=1.55 ; 95 \% \mathrm{CI}$ 0.71, 3.39; live birth model 4: $\mathrm{HR}=1 \cdot 36 ; 95 \%$ CI 0.59, 3.13; Table 4). Additionally, the associations between male AHEI-P score and achieving clinical pregnancy, live birth and pregnancy loss were examined. Results from the sensitivity analysis examining male AHEI-P as the exposure variable and the above pregnancy outcomes followed the same pattern as the results using female AHEI-P score (i.e. also non-significant; see online supplementary material, Supplemental Table S1).

\section{Discussion}

The present study is the first to examine dietary quality, as measured by the AHEI-P score, and achievement of clinical pregnancy. In this sample of couples planning their first pregnancy, females with greater adherence to the AHEI-P dietary pattern were more likely and more quickly able to achieve clinical pregnancy. Females who more closely followed the AHEI-P dietary pattern were also less likely to experience pregnancy loss. It would be prudent of us to acknowledge the wide and non-significant confidence intervals for these results (Table 4), but given the limited power, these analyses should be repeated with a larger sample size.

While there is a breadth of studies on nutritional factors and fertility, the discussion below focuses on examination of the literature on dietary patterns (i.e. a priori dietary quality scores or a posteriori dietary patterns), which may better reflect overall dietary intake more comprehensively than examination of single nutrients ${ }^{(13)}$. While sparse, these studies on likelihood of pregnancy and preconception dietary quality are most relevant to the current study. A study by Gaskins and colleagues examined the association between pre-pregnancy diet, using dietary indices, and pregnancy loss in women from the Nurses' Health Study II ${ }^{(20)}$. Dietary patterns, examined using a priori methods, were not significantly associated with pregnancy loss. It is worth noting that the present study included only nulliparous women, whereas the study by Gaskins et al. did not have this same criterion. Conversely, two other studies did find statistically significant relationships between preconception dietary patterns and likelihood of achieving 


\section{Public Health Nutrition}

Table 1 Alternative Healthy Eating Index for Pregnancy (AHEI-P) ${ }^{(8)}$ scoring method and mean (SD) AHEI-P scores for participants of the Lifestyle and Fertility (ISIS) study*

\begin{tabular}{|c|c|c|c|c|c|c|c|c|c|c|c|c|c|c|c|}
\hline \multirow[b]{2}{*}{ Component } & \multirow{2}{*}{$\begin{array}{l}\text { Criterion for minimum } \\
\text { score }(0)\end{array}$} & \multirow{2}{*}{$\begin{array}{l}\text { Criterion for maximum } \\
\text { score }(10)\end{array}$} & \multicolumn{2}{|c|}{ Score (all) } & \multicolumn{2}{|c|}{$\begin{array}{l}\text { Score for } \\
\text { females who } \\
\text { achieved } \\
\text { clinical } \\
\text { pregnancy } \\
(n 80) \\
\end{array}$} & \multicolumn{2}{|c|}{$\begin{array}{l}\text { Score for } \\
\text { females who } \\
\text { achieved } \\
\text { live birth } \\
(n 69)\end{array}$} & \multicolumn{2}{|c|}{$\begin{array}{l}\text { Score for } \\
\text { females who } \\
\text { achieved } \\
\text { clinical } \\
\text { pregnancy } \\
\text { but no live } \\
\text { birth }(n 11) \\
\end{array}$} & \multicolumn{2}{|c|}{$\begin{array}{c}\text { Score } \\
\text { (females) }\end{array}$} & \multicolumn{2}{|c|}{$\begin{array}{l}\text { Score } \\
\text { (males) }\end{array}$} & \multirow[b]{2}{*}{$P$ value } \\
\hline & & & Mean & SD & Mean & SD & Mean & SD & Mean & SD & Mean & SD & Mean & SD & \\
\hline Vegetables $\ddagger$ (servings/d) & 0 & $\geq 5$ & 5.5 & $2 \cdot 7$ & 5.5 & $2 \cdot 8$ & $5 \cdot 3$ & $2 \cdot 8$ & $6 \cdot 4$ & $2 \cdot 4$ & $5 \cdot 6$ & $2 \cdot 7$ & 5.5 & $2 \cdot 7$ & 0.82 \\
\hline Whole fruit§ (servings/d) & 0 & $\geq 4$ & 3.0 & 2.5 & $3 \cdot 2$ & 2.4 & $3 \cdot 2$ & $2 \cdot 2$ & $3 \cdot 7$ & 3.3 & $3 \cdot 4$ & 2.4 & $2 \cdot 6$ & 2.5 & 0.02 \\
\hline Whole grains $\|(g / d)$ & 0 & $\begin{array}{l}75 \text { (females) } \\
90 \text { (males) }\end{array}$ & $4 \cdot 2$ & $2 \cdot 7$ & 4.5 & $2 \cdot 7$ & 4.5 & $2 \cdot 8$ & $5 \cdot 0$ & $2 \cdot 3$ & $4 \cdot 3$ & $2 \cdot 6$ & $4 \cdot 0$ & $2 \cdot 9$ & 0.49 \\
\hline $\begin{array}{l}\text { Sugar-sweetened beverages } \\
\text { (servings/d) }\end{array}$ & $\geq 1$ & 0 & 4.4 & $4 \cdot 2$ & $5 \cdot 5$ & $3 \cdot 9$ & $5 \cdot 3$ & 3.9 & $6 \cdot 4$ & $3 \cdot 8$ & 4.9 & $4 \cdot 0$ & $3 \cdot 9$ & $4 \cdot 4$ & 0.05 \\
\hline Nuts and legumes ${ }^{\star *}$ (servings/d) & 0 & $\geq 1$ & $5 \cdot 7$ & 4.2 & $6 \cdot 1$ & 4.0 & $6 \cdot 1$ & $4 \cdot 0$ & 6.5 & 3.9 & $5 \cdot 8$ & $4 \cdot 1$ & 5.5 & 4.3 & 0.59 \\
\hline Red/processed meats†† (servings/d) & $\geq 1.5$ & $\overline{0}$ & 5.6 & $3 \cdot 6$ & $6 \cdot 6$ & $3 \cdot 1$ & $6 \cdot 6$ & 3.1 & $6 \cdot 8$ & $2 \cdot 9$ & $6 \cdot 6$ & $3 \cdot 1$ & 4.4 & $3 \cdot 7$ & $<0.0001$ \\
\hline 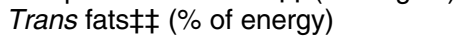 & $\geq 4$ & $\leq 0.5$ & $7 \cdot 1$ & 1.8 & 7.4 & $1 \cdot 6$ & 7.5 & 1.6 & $7 \cdot 1$ & 1.6 & 7.3 & 1.7 & $6 \cdot 8$ & 1.8 & 0.03 \\
\hline $\begin{array}{l}\text { Long-chain }(n-3) \text { fats } \\
\quad(\mathrm{EPA}+\mathrm{DHA}) \S \S(\mathrm{mg} / \mathrm{d})\end{array}$ & $\overline{0}$ & 250 & $3 \cdot 2$ & $3 \cdot 4$ & $1 \cdot 8$ & $2 \cdot 8$ & 1.9 & $2 \cdot 9$ & 1.5 & $1 \cdot 8$ & $2 \cdot 9$ & $3 \cdot 2$ & $3 \cdot 6$ & $3 \cdot 6$ & 0.06 \\
\hline PUFA || || (\% of energy) & $\leq 2$ & $\geq 10$ & $6 \cdot 6$ & 1.9 & 6.5 & $1 \cdot 8$ & $6 \cdot 6$ & 1.8 & $5 \cdot 8$ & 1.6 & $6 \cdot 6$ & 1.7 & $6 \cdot 7$ & $2 \cdot 0$ & 0.57 \\
\hline Naףी (mg/d) & $\begin{array}{l}\text { Highest decile: } \\
6287 \cdot 6 \text { (females) } \\
6900 \cdot 1 \text { (males) }\end{array}$ & $\begin{array}{l}\text { Lowest decile: } \\
1739 \cdot 6 \text { (females) } \\
2110 \cdot 1 \text { (males) }\end{array}$ & $5 \cdot 5$ & $2 \cdot 1$ & $5 \cdot 9$ & $1 \cdot 8$ & $6 \cdot 0$ & $1 \cdot 8$ & $5 \cdot 7$ & $1 \cdot 8$ & 5.9 & 1.9 & $5 \cdot 2$ & $2 \cdot 1$ & 0.004 \\
\hline $\mathrm{Ca}^{* \star *}(\mathrm{mg} / \mathrm{d})$ & 0 & $\geq 1200$ & $7 \cdot 0$ & $2 \cdot 1$ & $6 \cdot 9$ & $2 \cdot 0$ & $6 \cdot 8$ & 1.9 & $7 \cdot 4$ & $2 \cdot 3$ & $6 \cdot 8$ & $2 \cdot 0$ & $7 \cdot 3$ & $2 \cdot 2$ & 0.04 \\
\hline Folate†t† $(\mu \mathrm{g} / \mathrm{d})$ & 0 & $\geq 600$ & $7 \cdot 3$ & $2 \cdot 1$ & 6.9 & $2 \cdot 2$ & $6 \cdot 8$ & $2 \cdot 3$ & $7 \cdot 3$ & $2 \cdot 0$ & 6.9 & $2 \cdot 2$ & 7.8 & $2 \cdot 0$ & 0.0004 \\
\hline $\mathrm{Fe} \neq \ddagger \ddagger(\mathrm{mg} / \mathrm{d})$ & 0 & $\geq 27$ & $5 \cdot 8$ & 1.9 & $5 \cdot 1$ & 1.7 & 5.1 & 1.7 & $5 \cdot 7$ & $2 \cdot 1$ & $5 \cdot 2$ & 1.8 & $6 \cdot 3$ & 1.9 & $<0.0001$ \\
\hline AHEI-P & - & - & 69.8 & $14 \cdot 7$ & $72 \cdot 1$ & $13 \cdot 9$ & 71.6 & $14 \cdot 1$ & $75 \cdot 1$ & $12 \cdot 7$ & 71.0 & $13 \cdot 7$ & $68 \cdot 6$ & $15 \cdot 6$ & 0.19 \\
\hline
\end{tabular}

*Females, $n$ 132; males, $n 129$ (one male partner did not enrol in the study; two other males did not provide complete dietary data).

$\dagger P$ value for comparison between males and females using the $t$ test.

fIntake of $\geq 5$ servings of vegetables daily was considered optimal. One serving is 0.5 cup of vegetables or 1 cup of leafy green vegetables.

§Intake of $\geq 4$ servings of fruit daily was considered optimal. One serving is 1 medium piece of fruit or 0.5 cup of berries.

$\|$ Intake of $75 \mathrm{~g}$ of whole grains daily ( $\sim 5$ servings/d) for females and $90 \mathrm{~g} / \mathrm{d}(\sim 6$ servings/d) for males was considered optimal. One serving of $100 \%$ wholegrain product contains about $16 \mathrm{~g}$ whole grains (per dry weight).

IIntake of $\geq 1$ serving of sugar-sweetened beverages daily was considered to be the least optimal. Fruit juice, including $100 \%$ fruit juice, was included in this category. One serving is $237 \mathrm{ml}$ ( $8 \mathrm{fl}$. oz).

${ }^{*}$ Intake of $\geq 1$ serving of nuts and legumes daily was considered optimal. Meat alternatives such as tofu, tempeh, soya nuts and vegetable burgers were included in this category. One serving is $28 \mathrm{~g}(1 \mathrm{oz})$ of nuts or 1 tablespoon of peanut butter.

††Intake of $<1$ serving of red or processed meat monthly was considered optimal, with an upper limit of $\geq 1.5$ servings/d. One serving is $113 \mathrm{~g}(4 \mathrm{oz})$ of unprocessed meat or $43 \mathrm{~g}$ ( $1.5 \mathrm{oz}$ ) of processed meat.

+\#lntake of $\geq 4 \%$ of total energy intake from trans tats was considered least optimal.

\$S Intake of $250 \mathrm{mg}$ of EPA plus DHA daily $(\sim 2 \times 113 \mathrm{~g}(4 \mathrm{oz})$ servings of fish weekly) was considered optimal.

\|\| Intake of $\geq 10 \%$ of total energy intake from PUFA was considered optimal. PUFA does not include EPA or DHA intake.

Intake of $\geq 1200 \mathrm{mg} \mathrm{Ca} / \mathrm{d}$ was considered optimal.

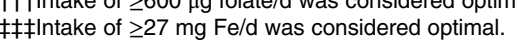




\section{Public Health Nutrition}

Table 2 Baseline characteristics of participants of the Lifestyle and Fertility (ISIS) study by female Alternative Healthy Eating Index for Pregnancy (AHEI-P) ${ }^{(8)}$ tertile scores (females, $n$ 132; males, $n$ 131)

\begin{tabular}{|c|c|c|c|c|c|c|c|c|c|}
\hline & \multicolumn{6}{|c|}{ AHEI-P score tertile, females (range) } & \multirow[b]{3}{*}{$P$ value* } & \multirow{2}{*}{\multicolumn{2}{|c|}{ Overall }} \\
\hline & \multicolumn{2}{|c|}{$\mathrm{T} 1(42 \cdot 5-65 \cdot 0)$} & \multicolumn{2}{|c|}{ T2 $(65 \cdot 2-78 \cdot 1)$} & \multicolumn{2}{|c|}{ T3 $(78 \cdot 6-102 \cdot 0)$} & & & \\
\hline & Mean or $n$ & SD or $\%$ & Mean or $n$ & SD or $\%$ & Mean or $n$ & SD or $\%$ & & Mean or $n$ & SD or $\%$ \\
\hline Female age (years), mean and SD & 29.2 & 3.4 & $29 \cdot 6$ & 3.2 & $30 \cdot 6$ & $3 \cdot 0$ & $0 \cdot 10$ & $29 \cdot 8$ & 3.2 \\
\hline Male age (years), mean and SD & 30.7 & 4.7 & $31 \cdot 1$ & $4 \cdot 3$ & $31 \cdot 8$ & 4.3 & 0.54 & $31 \cdot 2$ & 4.4 \\
\hline Female race/ethnicity, $n$ and $\%$ & & & & & & & 0.40 & & \\
\hline Non-Hispanic White & 34 & $25 \cdot 8$ & 30 & $22 \cdot 7$ & 36 & $27 \cdot 3$ & & 100 & $75 \cdot 8$ \\
\hline Non-Hispanic Black & 4 & 3.0 & 1 & 0.8 & 2 & 1.5 & & 7 & $5 \cdot 3$ \\
\hline Hispanic & 1 & 0.8 & 3 & $2 \cdot 3$ & 1 & 0.8 & & 5 & 3.8 \\
\hline Asian & 5 & 3.8 & 8 & $6 \cdot 1$ & 3 & $2 \cdot 3$ & & 16 & $12 \cdot 1$ \\
\hline Other/unknown & 0 & 0.0 & 2 & 1.5 & 2 & 1.5 & & 4 & 3.0 \\
\hline Male race/ethnicity $\dagger, n$ and $\%$ & & & & & & & 0.39 & & \\
\hline Non-Hispanic White & 37 & 28.5 & 30 & 23.1 & 38 & 29.2 & & 105 & 80.8 \\
\hline Non-Hispanic Black & 2 & 1.5 & 2 & 1.5 & 1 & 0.8 & & 5 & 3.9 \\
\hline Hispanic & 0 & 0.0 & 0 & 0.0 & 1 & 0.8 & & 1 & 0.8 \\
\hline Asian & 3 & $2 \cdot 3$ & 8 & $6 \cdot 2$ & 3 & $2 \cdot 3$ & & 14 & $10 \cdot 8$ \\
\hline Other/unknown & 1 & 0.8 & 3 & $2 \cdot 3$ & 1 & 0.8 & & 5 & 3.9 \\
\hline Female education, $n$ and $\%$ & & & & & & & 0.001 & & \\
\hline Less than college degree & 11 & 8.3 & 3 & $2 \cdot 3$ & 1 & 0.8 & & 15 & $11 \cdot 4$ \\
\hline College degree & 20 & $15 \cdot 2$ & 18 & 13.6 & 14 & $10 \cdot 6$ & & 52 & 39.4 \\
\hline Graduate degree & 13 & 9.9 & 23 & $17 \cdot 4$ & 29 & $22 \cdot 0$ & & 65 & 49.2 \\
\hline Male education, $n$ and $\%$ & & & & & & & 0.001 & & \\
\hline Less than college degree & 17 & $13 \cdot 0$ & 5 & 3.8 & 3 & $2 \cdot 3$ & & 25 & $19 \cdot 1$ \\
\hline College degree & 13 & 9.9 & 20 & $15 \cdot 3$ & 18 & $13 \cdot 7$ & & 51 & 38.9 \\
\hline Graduate degree & 13 & $9 \cdot 2$ & 19 & 14.5 & 23 & $17 \cdot 6$ & & 55 & $42 \cdot 0$ \\
\hline Annual household income $\neq$ (\$US), $n$ and \% & & & & & & & 0.82 & & \\
\hline$<60000$ & 14 & $10 \cdot 9$ & 12 & $9 \cdot 3$ & 9 & $7 \cdot 0$ & & 35 & $27 \cdot 1$ \\
\hline $60000-99999$ & 13 & $10 \cdot 1$ & 13 & $10 \cdot 1$ & 15 & 11.6 & & 41 & 31.8 \\
\hline$\geq 100000$ & 16 & $12 \cdot 4$ & 19 & 14.7 & 18 & 14.0 & & 53 & $41 \cdot 1$ \\
\hline Female smoking status, $n$ and $\%$ & & & & & & & 0.67 & & \\
\hline Never & 33 & $25 \cdot 0$ & 36 & $27 \cdot 3$ & 35 & $26 \cdot 5$ & & 104 & 78.8 \\
\hline Past use & 10 & 7.6 & 8 & $6 \cdot 1$ & 9 & $6 \cdot 8$ & & 27 & 20.5 \\
\hline Current use & 1 & 0.8 & 0 & 0.0 & 0 & 0.0 & & 1 & 0.8 \\
\hline Male smoking status, $n$ and $\%$ & & & & & & & 0.01 & & \\
\hline Never & 24 & 18.5 & 27 & 20.8 & 20 & 15.4 & & 71 & 54.6 \\
\hline Past use & 11 & 8.5 & 16 & $12 \cdot 3$ & 23 & $17 \cdot 7$ & & 50 & 38.5 \\
\hline Current use & 7 & 5.4 & 1 & 0.8 & 1 & 0.8 & & 9 & 6.9 \\
\hline
\end{tabular}




\section{Public Health Nutrition}

Table 2 Continued

\begin{tabular}{|c|c|c|c|c|c|c|c|c|c|}
\hline & \multicolumn{6}{|c|}{ AHEI-P score tertile, females (range) } & \multirow[b]{3}{*}{$P$ value* $^{*}$} & \multirow{2}{*}{\multicolumn{2}{|c|}{ Overall }} \\
\hline & \multicolumn{2}{|c|}{ T1 (42.5-65.0) } & \multicolumn{2}{|c|}{ T2 $(65 \cdot 2-78 \cdot 1)$} & \multicolumn{2}{|c|}{ T3 $(78 \cdot 6-102 \cdot 0)$} & & & \\
\hline & Mean or $n$ & SD or $\%$ & Mean or $n$ & SD or $\%$ & Mean or $n$ & SD or $\%$ & & Mean or $n$ & SD or $\%$ \\
\hline Female alcohol intake $(\mathrm{g} / \mathrm{d})$, mean and SD & 4.6 & 9.6 & $7 \cdot 3$ & $11 \cdot 2$ & $5 \cdot 6$ & $9 \cdot 3$ & 0.46 & 5.9 & $10 \cdot 1$ \\
\hline Male alcohol intake $(\mathrm{g} / \mathrm{d})$, mean and SD & 13.1 & $20 \cdot 3$ & $9 \cdot 8$ & $15 \cdot 4$ & 4.8 & 8.9 & 0.05 & $9 \cdot 2$ & $15 \cdot 8$ \\
\hline Female caffeine intake $(\mathrm{mg} / \mathrm{d})$, mean and SD & 61.5 & $66 \cdot 4$ & 83.9 & 71.9 & $98 \cdot 6$ & $91 \cdot 1$ & 0.08 & $81 \cdot 3$ & $78 \cdot 1$ \\
\hline Female aerobic activity, $n$ and $\%$ & & & & & & & $0 \cdot 15$ & & \\
\hline$<30 \mathrm{~min} /$ week & 11 & $8 \cdot 3$ & 6 & $2 \cdot 3$ & 3 & $2 \cdot 3$ & & 20 & $15 \cdot 2$ \\
\hline 30-59 min/week & 12 & $9 \cdot 1$ & 10 & $7 \cdot 6$ & 9 & $6 \cdot 8$ & & 31 & 23.5 \\
\hline $1-2 \mathrm{~h}$ week & 8 & $6 \cdot 1$ & 8 & $6 \cdot 1$ & 13 & $9 \cdot 9$ & & 31 & 23.5 \\
\hline$\geq 3 \mathrm{~h} /$ week & 11 & $8 \cdot 3$ & 20 & $15 \cdot 2$ & 19 & 14.4 & & 50 & 37.9 \\
\hline Regular menstrual cycles§, $n$ and \% & & & & & & & 1.00 & & \\
\hline No & 3 & $2 \cdot 3$ & 3 & $2 \cdot 3$ & 3 & $2 \cdot 3$ & & 9 & $7 \cdot 0$ \\
\hline Yes & 39 & 30.2 & 40 & $31 \cdot 0$ & 41 & 31.8 & & 120 & 93.0 \\
\hline Age at menarche (years), mean and SD & $12 \cdot 8$ & 1.4 & $13 \cdot 0$ & 1.4 & $12 \cdot 7$ & 1.5 & 0.71 & $12 \cdot 8$ & 1.4 \\
\hline Female pre-pregnancy BMI $\left(\mathrm{kg} / \mathrm{m}^{2}\right)$, mean and SD & $26 \cdot 8$ & $6 \cdot 5$ & $23 \cdot 0$ & $4 \cdot 3$ & $23 \cdot 1$ & $4 \cdot 1$ & $<0.001$ & $24 \cdot 3$ & $5 \cdot 4$ \\
\hline Male BMl $\left(\mathrm{kg} / \mathrm{m}^{2}\right)$, mean and SD & 29.5 & $5 \cdot 0$ & $26 \cdot 1$ & $4 \cdot 1$ & $27 \cdot 5$ & $7 \cdot 3$ & 0.02 & $27 \cdot 7$ & $5 \cdot 8$ \\
\hline Female total energy intake $(\mathrm{kJ} / \mathrm{d})$, mean and SD & $6875 \cdot 1$ & $2051 \cdot 4$ & $7091 \cdot 0$ & $1804 \cdot 6$ & $7702 \cdot 7$ & 2081.5 & 0.13 & $7222 \cdot 8$ & $1999 \cdot 1$ \\
\hline Female total energy intake $(\mathrm{kcal} / \mathrm{d})$, mean and SD & $1643 \cdot 2$ & $490 \cdot 3$ & $1694 \cdot 8$ & $431 \cdot 3$ & $1841 \cdot 0$ & $497 \cdot 5$ & 0.13 & $1726 \cdot 3$ & $477 \cdot 8$ \\
\hline Male total energy intake $\|(\mathrm{kJ} / \mathrm{d})$, mean and SD & 8437.0 & $2760 \cdot 6$ & $9215 \cdot 7$ & 2541.4 & 9545.4 & $2420 \cdot 9$ & 0.13 & $9072 \cdot 2$ & 2597.8 \\
\hline Male total energy intake $\|(\mathrm{kcal} / \mathrm{d})$, mean and SD & $2016 \cdot 5$ & 659.8 & $2202 \cdot 6$ & $607 \cdot 4$ & 2281.4 & $578 \cdot 6$ & $0 \cdot 13$ & $2168 \cdot 3$ & $620 \cdot 9$ \\
\hline Female AHEI-P score, mean and SD & 55.5 & $6 \cdot 2$ & $71 \cdot 0$ & $4 \cdot 1$ & $86 \cdot 4$ & $5 \cdot 6$ & $<0.0001$ & $72 \cdot 0$ & $13 \cdot 8$ \\
\hline Range & $42 \cdot 5$ & & $65 \cdot 2-$ & & $78 \cdot 6-$ & $2 \cdot 0$ & & & \\
\hline $\begin{array}{l}\text { Male AHEI-P score } \| \text {, mean and SD } \\
\text { Range }\end{array}$ & $\begin{array}{l}51 \cdot 5 \\
35 \cdot 3\end{array}$ & $.8^{6.5}$ & $\begin{array}{l}67 \cdot 6 \\
61 \cdot 2\end{array}$ & $5 \cdot 6^{4 \cdot 1}$ & $\begin{array}{l}86 \cdot 6 \\
76 \cdot 8\end{array}$ & $1 \cdot 1^{7 \cdot 8}$ & $<0.0001$ & $68 \cdot 7$ & $15 \cdot 6$ \\
\hline
\end{tabular}

${ }^{*} P$ value for comparison across AHEI-P tertiles using ANOVA (continuous variables) and the $\chi^{2}$ and Fisher's exact tests (categorical variables).

tOne participant was missing ethnicity.

†Three couples who reported unknown incomes were excluded from analysis.

§Three females were missing information on regularity of menstrual cycles at baseline.

$\|$ Two male participants did not have complete dietary data; therefore, $n 129$ for total energy and AHEI-P component scores and total score. 
Table 3 Clinical pregnancy status by Alternative Healthy Eating Index for Pregnancy (AHEI-P) $)^{(8)}$ score tertile for females of the Lifestyle and Fertility (ISIS) study $(n 132)^{\star}$

\begin{tabular}{|c|c|c|c|c|c|c|}
\hline \multirow{3}{*}{$\begin{array}{l}\text { Achieved clinical } \\
\text { pregnancy }\end{array}$} & \multicolumn{6}{|c|}{ AHEI-P tertile (range) } \\
\hline & \multicolumn{2}{|c|}{$\begin{array}{c}\text { Low } \\
(42 \cdot 5-65 \cdot 0)\end{array}$} & \multicolumn{2}{|c|}{$\begin{array}{l}\text { Medium } \\
(65 \cdot 2-78 \cdot 1)\end{array}$} & \multicolumn{2}{|c|}{$\begin{array}{c}\text { High } \\
(78.6-102.0)\end{array}$} \\
\hline & $\%$ & $n$ & $\%$ & $n$ & $\%$ & $n$ \\
\hline No & 40.9 & 18 & $45 \cdot 5$ & 20 & $31 \cdot 8$ & 14 \\
\hline Yes & $59 \cdot 1$ & 26 & $54 \cdot 6$ & 24 & $68 \cdot 2$ & 30 \\
\hline
\end{tabular}

${ }^{*} P$ value $=0.41$ determined by $\chi^{2}$ test.

Table 4 Cox proportional hazards regression analysis for time to pregnancy as a function of Alternative Healthy Eating Index for Pregnancy (AHEI-P) ${ }^{(8)}$ score (tertile) and selected covariates for couples participating in the Lifestyle and Fertility (ISIS) study ( $n$ 117). Hazard ratio (HR) with $95 \% \mathrm{Cl}$ for time to pregnancy outcomes*

\begin{tabular}{|c|c|c|c|}
\hline & HR & $95 \% \mathrm{Cl}$ & $P$ value \\
\hline \multicolumn{4}{|c|}{ Clinical pregnancy $(n 80)$} \\
\hline \multicolumn{4}{|l|}{ Model $1 \dagger$} \\
\hline AHEI-P medium & 1.23 & $0.67,2.27$ & 0.50 \\
\hline AHEI-P high & $1 \cdot 20$ & $0.66,2.17$ & 0.56 \\
\hline \multicolumn{4}{|l|}{ Model $2 \ddagger$} \\
\hline AHEI-P medium & 1.34 & $0.70,2.53$ & 0.38 \\
\hline AHEI-P high & 1.25 & $0.66,2.35$ & 0.49 \\
\hline \multicolumn{4}{|l|}{ Model 3§ } \\
\hline AHEI-P medium & 1.50 & $0.72,3.14$ & 0.28 \\
\hline AHEI-P high & 1.43 & $0.68,2.95$ & 0.35 \\
\hline \multicolumn{4}{|l|}{ Model $4 \|$} \\
\hline AHEI-P medium & 1.64 & $0.78,3.47$ & 0.19 \\
\hline AHEI-P high & 1.55 & $0.71,3.39$ & 0.27 \\
\hline \multicolumn{4}{|c|}{ Clinical pregnancy that resulted in live birth ( $n 69)$} \\
\hline \multicolumn{4}{|c|}{ Model 15} \\
\hline AHEI-P medium & 1.35 & $0.70,2.59$ & 0.37 \\
\hline AHEI-P high & 1.14 & $0.59,2.20$ & 0.70 \\
\hline \multicolumn{4}{|l|}{ Model $2 \ddagger$} \\
\hline AHEI-P medium & 1.46 & $0.74,2.90$ & 0.28 \\
\hline AHEI-P high & 1.19 & $0.59,2.38$ & 0.63 \\
\hline \multicolumn{4}{|l|}{ Model $3 \S$} \\
\hline AHEI-P medium & 1.48 & $0.68,3.21$ & 0.32 \\
\hline AHEI-P high & 1.23 & $0.56,2.69$ & 0.60 \\
\hline \multicolumn{4}{|l|}{ Model $4 \|$} \\
\hline AHEI-P medium & 1.63 & $0.74,3.59$ & 0.22 \\
\hline AHEI-P high & 1.36 & $0.59,3.13$ & 0.46 \\
\hline \multicolumn{4}{|l|}{ Pregnancy loss ( $n$ 11) } \\
\hline \multicolumn{4}{|l|}{ Model $1 \dagger$} \\
\hline AHEI-P medium & 1.02 & $0.16,6.56$ & 0.99 \\
\hline AHEI-P high & 0.65 & $0.14,3.12$ & 0.59 \\
\hline \multicolumn{4}{|l|}{ Model $2 \ddagger$} \\
\hline AHEI-P medium & 0.99 & $0.15,6.50$ & 0.99 \\
\hline AHEI-P high & 0.55 & $0.10,3.02$ & 0.49 \\
\hline \multicolumn{4}{|l|}{ Model 3§ } \\
\hline AHEI-P medium & $2 \cdot 72$ & $0.26,28.92$ & 0.41 \\
\hline AHEI-P high & 1.25 & $0.13,12.32$ & 0.85 \\
\hline \multicolumn{4}{|l|}{ Model $4 \|$} \\
\hline AHEI-P medium & $2 \cdot 31$ & $0.21,25.89$ & 0.50 \\
\hline AHEI-P high & 0.93 & $0.09,10.22$ & 0.95 \\
\hline
\end{tabular}

*The sample size does not include the $n 15$ who were censored at baseline visit. All covariates are for the female unless otherwise indicated. The reference group is the lowest tertile of AHEI-P. P values determined by Cox proportional hazards regression.

†Adjusted for age and energy intake.

$\ddagger$ Adjusted for age, energy intake and BMI.

§Adjusted for age, energy intake, BMI, male education and male smoking status.

|| Adjusted for age, energy intake, BMI, male education, male smoking status and male AHEI-P score.

pregnancy; however, results may not be directly comparable as these studies used couples undergoing assisted reproductive technology ${ }^{(21,22)}$.
While the current study is unique in that it is the first to examine AHEI-P score and achieving clinical pregnancy, studies have utilized other methods to characterize 
dietary patterns in relation to pregnancy outcomes. Chavarro et al. described the 'fertility diet' pattern, which was characterized by high intakes of monounsaturated to trans fats, high-fat dairy and Fe, higher frequency of multivitamin use, lower glycaemic load and lower intakes of animal protein and low-fat dairy ${ }^{(23)}$. Females with greater adherence to the 'fertility diet' associated with a $69 \%$ lower risk of ovulatory disorder infertility and this relationship was not modified by differences in age, parity or BMI. Although a direct comparison between the AHEI-P and the 'fertility diet' score is difficult, results from the present study are similar to those of Charvarro and colleagues in that those who achieved a clinical pregnancy had higher diet quality (i.e. higher mean (SD) AHEI-P score: 72.1 (13.9); $n$ 80) compared with those who did not achieve clinical pregnancy (69.3 (SD 13.4); $n$ 52; $P<0.001$; data not shown). Similarities between the AHEI-P and the 'fertility diet' score can be seen in that both award higher points to diets that are rich in vegetables, healthy fats and $\mathrm{Fe}$, and low in animal protein/red/processed meats.

With regard to pregnancy loss, Maconochie et al. examined associations between biological, behavioural and lifestyle risk factors, including nutrition, and found that fresh fruit and vegetable intake on most days decreased the odds of having a miscarriage by half ${ }^{(24)}$. Additionally, consumption of dairy products and chocolate, and eating fish or white meat twice weekly were also suggestive of protective effects against miscarriage. Interestingly, both increasing frequency and average weekly intake of alcohol were found to be associated with a statistically significant increased risk of miscarriage ${ }^{(24)}$. Similarly, in other study conducted in Italy that examined pregnancy loss, alcohol consumption before pregnancy was also associated with increased risk of spontaneous abortion. In that same study, consumption of green vegetables, fruit, milk, cheese, fish and eggs was also found to be protective ${ }^{(25)}$. It is noteworthy that the AHEI-P score used in the current study does not include alcohol or caffeine as components of the score. We evaluated these variables in our analyses; including them in our multivariate models did not appreciably change our results.

The current study is distinct in that it is the first to examine AHEI-P score and pregnancy outcomes using pre-pregnancy diet in nulliparous couples. Three other studies have utilized the AHEI-P to investigate dietary quality related to pregnancy outcomes; however, the outcomes have been related to infant birth weight and did not examine the likelihood of achieving pregnancy, and therefore are not addressed in the present paper ${ }^{(17,26,27)}$.

Results of the current study should be interpreted in the context of its strengths and limitations. It is the first study to examine dietary quality by AHEI-P in a sample of nulliparous couples planning pregnancy. The current study is also unique in that it accounts for male dietary quality in terms of pregnancy outcomes. It is worth noting that the sample size was modest, particularly for the analysis that examined those who experienced pregnancy loss ( $n$ 11) and may have been too small to detect significance. Therefore, interpretation of these findings must be made with caution. However, the study is exploratory in its use of the AHEI$\mathrm{P}$ with male dietary data. There were statistically significant differences in some components of the AHEI-P between males and females; however, there were no differences in total AHEI-P between males and females. Thus, the AHEI-P may not capture all dietary components that may be relevant to the likelihood of pregnancy. The AHEI-P was developed for females and it is unknown how relevant the AHEI-P is for males. The AHEI-P may not adequately capture components of the diet that may affect male fertility (i.e. sperm count and quality), like fish intake ${ }^{(28)}$ or caffeine ${ }^{(29)}$. Nevertheless, it is interesting that the relationship between maternal dietary quality and likelihood of achieving pregnancy increased once paternal dietary quality was included in the model (Table 4: models 3 and 4). In Table 4, model 4 adjusts for all the variables from model 3, plus the addition of male AHEI-P score. The HR increased from 1.50 to 1.64 (AHEI-P medium) and from 1.43 to 1.55 (AHEI-P high) when comparing model 3 with model 4 . While these results are not statistically significant, the magnitude of the HR increases. While less is known about the role that paternal nutrition plays in reproduction, results suggest that higher male dietary quality may be related to increased chances of achieving pregnancy. Dietary intakes of couples can be correlated; however, in this sample, correlations between the components of the AHEI-P were moderate. For example, the Spearman correlation for total AHEI-P score between males and females was $0.52(P<0.001)$.

\section{Conclusions}

In conclusion, results suggest that higher pre-pregnancy dietary quality, as measured by the AHEI-P, was associated with increased likelihood of achieving pregnancy, including pregnancy that resulted in live birth. Adherence to the AHEI$\mathrm{P}$ dietary pattern was also associated with a decreased risk of pregnancy loss. The role of pre-pregnancy diet in fertility is an area that deserves continued research. Future studies should address these research questions with adequate power. While the link between nutrition before pregnancy and pregnancy outcomes has been studied, it is possible that there are dietary components that are important for increasing the likelihood of getting pregnant that are not captured in the AHEI-P. Additionally, the AHEI-P has not been assessed for validity and reliability. Future studies should consider the development and evaluation of a dietary quality score that more accurately measures the likelihood of achieving pregnancy. Determining components of a dietary pattern that supports achieving conception is a high priority for future research. Because diet is a modifiable risk factor, examining preconception diet to identify nutrients important in achieving conception may be a potential approach to use with females planning pregnancy. 


\section{Acknowledgements}

Financial support: Research reported in this publication was supported by the Eunice Kennedy Shriver National Institute of Child Health \& Human Development of the National Institutes of Health (award numbers R01HD049762 and 3R01HD049762S1). Partial support was provided by the Clinical and Translational Science Institute (General Clinical Research Center) at Penn State University (grant number NIH M01 107 32). The content is solely the responsibility of the authors and does not necessarily represent the official views of the National Institutes of Health. The funders had no role in the design, analysis or writing of this article. Conflict of interest: None. Authorship: All authors participated in the study concept, design, analysis, editing and revision of the manuscript. Ethics of human subject participation: This study was conducted according to the guidelines laid down in the Declaration of Helsinki and all procedures involving human subjects/patients were approved by the United States-Boston IVF, The Pennsylvania State University and Dartmouth-Hitchcock Medical Center Institutional Review Boards. Written informed consent was obtained from all subjects/patients.

\section{Supplementary material}

To view supplementary material for this article, please visit https://doi.org/10.1017/S1368980019001290

\section{References}

1. Sharma R, Biedenharn KR, Fedor JM et al. (2013) Lifestyle factors and reproductive health: taking control of your fertility. Reprod Biol Endocrinol 11, 66.

2. Peck JD, Leviton A \& Cowan LD (2010) A review of the epidemiologic evidence concerning the reproductive health effects of caffeine consumption: a 2000-2009 update. Food Chem Toxicol 48, 2549-2576.

3. Gaskins AJ \& Chavarro JE (2018) Diet and fertility: a review. Am J Obstet Gynecol 218, 379-389.

4. Arab A, Rafie N, Mansourian M et al. (2018) Dietary patterns and semen quality: a systematic review and meta-analysis of observational studies. Andrology 6, 20-28.

5. Chavarro JE (2017) Healthy diets and men's contribution to fertility; is semen quality good enough? Fertil Steril 107, 906-907.

6. Nassan FL, Chavarro JE \& Tanrikut C (2018) Diet and men's fertility: does diet affect sperm quality? Fertil Steril 110, 570-577.

7. Efrat M, Stein A, Pinkas H et al. (2018) Dietary patterns are positively associated with semen quality. Fertil Steril 109, 809-816.

8. Mendiola J, Torres-Cantero AM, Vioque J et al. (2010) A low intake of antioxidant nutrients is associated with poor semen quality in patients attending fertility clinics. Fertil Steril $\mathbf{9 3}$, 1128-1133.

9. Wong WY, Zielhuis GA, Thomas CM et al. (2003) New evidence of the influence of exogenous and endogenous factors on sperm count in man. Eur J Obstet Gynecol Reprod Biol 110, 49-54.

10. Attaman JA, Toth TL, Furtado J et al. (2012) Dietary fat and semen quality among men attending a fertility clinic. Hum Reprod 27, 1466-1474.
11. Chavarro JE, Furtado J, Toth TL et al. (2011) Trans-fatty acid levels in sperm are associated with sperm concentration among men from an infertility clinic. Fertil Steril 95, 1794-1797.

12. Muthusami KR \& Chinnaswamy P (2005) Effect of chronic alcoholism on male fertility hormones and semen quality. Fertil Steril 84, 919-924.

13. Hu FB (2002) Dietary pattern analysis: a new direction in nutritional epidemiology. Curr Opin Lipidol 13, 3-9.

14. Kennedy ET, Ohls J, Carlson S et al. (1995) The Healthy Eating Index: design and applications. J Am Diet Assoc 95, 1103-1108.

15. McCullough ML, Feskanich D, Stampfer MJ et al. (2002) Diet quality and major chronic disease risk in men and women: moving toward improved dietary guidance. Am J Clin Nutr 76, 1261-1271.

16. Chiuve SE, Fung TT, Rimm EB et al. (2012) Alternative dietary indices both strongly predict risk of chronic disease. J Nutr 142, 1009-1018.

17. Poon AK, Yeung E, Boghossian N et al. (2013) Maternal dietary patterns during third trimester in association with birthweight characteristics and early infant growth. Scientifica 2013, 786409.

18. Jonnalagadda SS, Mitchell DC, Smiciklas-Wright $\mathrm{H}$ et al. (2000) Accuracy of energy intake data estimated by a multiple-pass, 24-hour dietary recall technique. J Am Diet Assoc 100, 303-308.

19. Monden CWS, van Lenthe F, De Graaf NDD et al. (2003) Partner's and own education: does who you live with matter for self-assessed health, smoking and excessive alcohol consumption? Soc Sci Med 57, 1901-1912.

20. Gaskins AJ, Rich-Edwards JW, Hauser R et al. (2014) Prepregnancy dietary patterns and risk of pregnancy loss. Am J Clin Nutr 100, 1166-1172.

21. Twigt JM, Bolhuis ME, Steegers EA et al. (2012) The preconception diet is associated with the chance of ongoing pregnancy in women undergoing IVF/ICSI treatment. Hum Reprod 27, 2526-2531.

22. Vujkovic M, de Vries JH, Lindemans J et al. (2010) The preconception Mediterranean dietary pattern in couples undergoing in vitro fertilization/intracytoplasmic sperm injection treatment increases the chance of pregnancy. Fertil Steril 94, 2096-2101.

23. Chavarro JE, Rich-Edwards JW, Rosner BA et al. (2007) Diet and lifestyle in the prevention of ovulatory disorder infertility. Obstet Gynecol 110, 1050-1058.

24. Maconochie N, Doyle P, Prior S et al. (2007) Risk factors for first trimester miscarriage - results from a UK-populationbased case-control study. BJOG 114, 170-186.

25. Di Cintio E, Parazzini F, Chatenoud L et al. (2001) Dietary factors and risk of spontaneous abortion. Eur J Obstet Gynecol Reprod Biol 95, 132-136.

26. Rodriguez-Bernal CL, Rebagliato M, Iniguez C et al. (2010) Diet quality in early pregnancy and its effects on fetal growth outcomes: the Infancia y Medio Ambiente (Childhood and Environment) Mother and Child Cohort Study in Spain. Am J Clin Nutr 91, 1659-1666.

27. Rifas-Shiman SL, Rich-Edwards JW, Kleinman KP et al. (2009) Dietary quality during pregnancy varies by maternal characteristics in Project Viva: a US cohort. J Am Diet Assoc 109, 1004-1011.

28. Afeiche MC, Gaskins AJ, Williams PL et al. (2014) Processed meat intake is unfavorably and fish intake favorably associated with semen quality indicators among men attending a fertility clinic. J Nutr 144, 1091-1098.

29. Jensen TK, Swan SH, Skakkebaek NE et al. (2010) Caffeine intake and semen quality in a population of 2,554 young Danish men. Am J Epidemiol 171, 883-891. 